\title{
Light with finite rotation - an attempt for a theoretical description
}

\author{
Hanson, Steen Grüner
}

Published in:

Proceedings of SPIE

Link to article, DOI:

$10.1117 / 12.2319872$

Publication date:

2018

Document Version

Publisher's PDF, also known as Version of record

Link back to DTU Orbit

Citation (APA):

Hanson, S. G. (2018). Light with finite rotation - an attempt for a theoretical description. In Proceedings of SPIE (Vol. 10834). [108340F ] SPIE - International Society for Optical Engineering. Proceedings of SPIE - The International Society for Optical Engineering https://doi.org/10.1117/12.2319872

\section{General rights}

Copyright and moral rights for the publications made accessible in the public portal are retained by the authors and/or other copyright owners and it is a condition of accessing publications that users recognise and abide by the legal requirements associated with these rights.

- Users may download and print one copy of any publication from the public portal for the purpose of private study or research.

- You may not further distribute the material or use it for any profit-making activity or commercial gain

- You may freely distribute the URL identifying the publication in the public portal

If you believe that this document breaches copyright please contact us providing details, and we will remove access to the work immediately and investigate your claim 


\section{Light with finite rotation: an attempt for a theoretical description}

\section{Steen G. Hanson}

Steen G. Hanson, "Light with finite rotation: an attempt for a theoretical description," Proc. SPIE 10834, Speckle 2018: VII International Conference on Speckle Metrology, 108340F (7 September 2018); doi:

10.1117/12.2319872

SPIE Event: SPECKLE 2018: VII International Conference on Speckle Metrology, 2018, Janów Podlaski, Poland 


\title{
Light with finite rotation - an attempt for a theoretical description
}

\author{
Steen G. Hanson, Department of Photonics Engineering, Technical University of \\ Denmark, Denmark ${ }^{1}$
}

\begin{abstract}
:
The metamaterial filter introduced in the form of the so-called METATOY Johannes Courtial et al., j. opt. 13 (2011))[1] has a series of interesting properties, although it can presently only be realized as an array of discrete elements, being it an array of Dove prisms or lens arrays. Nevertheless, a theoretical analysis of field propagation through such a twisting filter is still lacking. Based on the complex-valued ray matrix formalism (ABCDmatrices/canonical transforms), the propagation through such a filter can be mimicked by using the known Green's function (e.g. Aykut et al. Journal of the Optical Society of America a (2010) 27(9) 1896)[2]. This matrix for an entire system including a flipping filter is non-symplectic, which in fact indicates that this filter's perturbation is rayoptically forbidden. However, if we proceed and insert the matrix-values into this Green's function, we arrive at results that are in agreement with the previously shown examples with METATOY. It is further shown how e.g. Fourier transformation of this filter will give rise to unexpected ray transformations. Finally, a new ray-optically forbidden element is discussed, as will possible future applications.
\end{abstract}

Keywords: Metamaterial, Canonical Transforms, Matrix Optics.

\section{INTRODUCTION}

The use of ray matrices for description of ray propagation - and field propagation, as well - has been commonly used for analyzing propagation through optical elements with transverse phase variations up to second order, i.e. lenses, free space and curved mirrors [3], all applicable within the Fresnel region. Later, the introduction of Gaussian shaped apertures were introduced giving rise to complex valued entries in the ray matrices [4],[5]. This has opened up for deriving analytical results within speckle-based sensors, interferometry, and optical coherence tomography, just to mention a few.

In the following, we will first address the possibilities and restrictions for the ray transfer matrices used up until now. Then will follow a presentation of the light-ray-direction-flipping plate, named METATOY [1], [6],[7] and [8] and hereafter named the flipping filter. The crux of this presentation will be the introduction of a non-symplectic matrix to describe the METATOY plate. Following this, we will apply the matrix elements in the Green's function and give some examples of incoherent and coherent propagation through an optical system that includes the rayflipping filter.

Finally, we will introduce a new optical -forbidden - element that flips the light direction based on its incident position. A lens does this, but here the optical element flips the light azimuthally. Again, we will provide a matrix description for the element.

\footnotetext{
${ }^{1}$ vsgh@fotonik.dtu.dk
} 


\section{SYMPLECTIC MATRICES FOR SECOND-ORDER OPTICAL ELEMENTS}

The general matrix for 2-D optical systems in paraxial systems is given by:

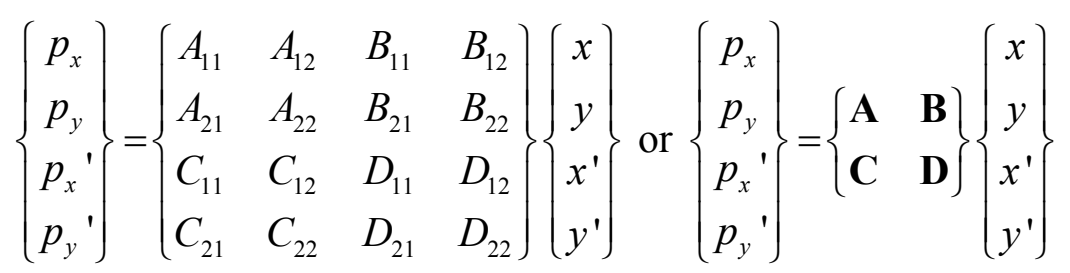

Here the input coordinates and input slopes are $(x, y)$ and $\left(x^{\prime}, y^{\prime}\right)$, respectively, and the corresponding coordinates in the output plane are $\left(p_{x}, p_{y}\right)$ and $\left(p_{x}, p_{y}{ }^{\prime}\right)$. Thus, the submatrices act as follows:

- A is a 2x2 matrix giving the relation between output and input ray positions, i.e. the "magnification" of the optical system

- B is a $2 \times 2$ matrix governing the relation between output position and input slope, i.e. the "length" of the optical system. Exact imaging usually requires the real part of this element to vanish.

- C is a $2 \times 2$ matrix relating the output slope with the input position, i.e. an "anamorphic lens", or in case the matrix elements are complex, a tilted Gaussian aperture.

- D is a $2 \times 2$ matrix that relates the output and input slopes.

When we deal with a $4 \times 4$ ray tranfer matrix, we have included optically elements that are not aligned with the principal axes.

The four most commonly used matrices are:

The anamorphic lens: $\quad \mathbf{M}$ lens $=\left\{\begin{array}{cccc}1 & 0 & 0 & 0 \\ 0 & 1 & 0 & 0 \\ -1 / f_{x} & 0 & 1 & 0 \\ 0 & -1 / f_{y} & 0 & 1\end{array}\right\}$

If the lens was tilted with respect to the axes, the $\mathbf{C}$-matrix would have real-valued off-diagonal elements.

$$
\begin{aligned}
& \text { Free space propagation a distance } L: \quad \mathbf{M}_{\text {freespace }}=\left\{\begin{array}{cccc}
1 & 0 & L & 0 \\
0 & 1 & 0 & L \\
0 & 0 & 1 & 0 \\
0 & 0 & 0 & 1
\end{array}\right\} \\
& \text { An assymetric Gaussian aperture: } \mathbf{M}_{\text {aperture }}=\left\{\begin{array}{cccc}
1 & 0 & 0 & 0 \\
0 & 1 & 0 & 0 \\
-2 i / k \omega_{x}^{2} & 0 & 1 & 0 \\
0 & -2 i / k \omega_{y}^{2} & 0 & 1
\end{array}\right\}
\end{aligned}
$$

Here, complex-valued off-diagonal elements would appear if the aperture was not aligned with the axes. 
All the above matrices will have unit determinant, and so will the matrix arising when a succession of matrices are multiplied in order to characterize an entire optical system with only one matrix. Note that some articles erroneously state that absorption gives rise to the determinant being different from unity! The requirement that the determinant is unity means that a $4 \times 4$ matrix has 10 degrees of freedom, or independent variables. In case of a $2 \times 2$ matrix describing a symmetric system, three independent variables exist if the input and output medium have the same refractive index. If we derive the matrix for a succession of two identical optical elements, we get the expected combined effect.

What in connection with this paper is more important is the requirement that the matrices for the above optical systems have to be symplectic [3] in order for the eikonal to follow Fermat's principle. This implies that for any optical matrix $\mathbf{M}$ the following relation must hold:

$$
\mathbf{M} \boldsymbol{\Omega} \mathbf{M}^{T}=\boldsymbol{\Omega},
$$

where $\boldsymbol{\Omega}=\left\{\begin{array}{cc}\mathbf{0} & \mathbf{1} \\ -1 & 0\end{array}\right\}$. And for the mentioned matrices, these all are symplectic matrices belonging to the symplectic Lie group $\mathrm{SL}_{2}(\mathbf{R})$, and if we include the matrix for the aperture, the group is expanded to $\mathrm{SL}_{2}(\mathbf{C})$. Thus, a multiplication of such matrices will give a new matrix within this group. Should an optical system not obey Fermat's principle, its associated matrix - if it exists - will not be symplectic.

\section{MATRIX FOR THE LIGHT-RAY-DIRECTION-FLIPPING PLATE}

We now follow the descriptions given for the light-ray-direction-flipping plate named METATOY in [6], [7], and [8].

We consider a plate that acts on an incident ray having an in-plane direction $\left(x^{\prime}, y^{\prime}\right)$. The outgoing ray will, by virtue of this plate, obtain a directional change $\theta$ causing the outgoing direction $\left(p_{x}{ }^{\prime}, p_{y}{ }^{\prime}\right)$ to become:

$$
\left(p_{x}{ }^{\prime}, p_{y}{ }^{\prime}\right)=\left(x^{\prime} \cos [\theta]+y^{\prime} \sin [\theta], y^{\prime} \cos [\theta]-x^{\prime} \sin [\theta]\right)
$$

or in matrix form:

$$
\left\{\begin{array}{c}
p_{x}{ }^{\prime} \\
p_{y}^{\prime}
\end{array}\right\}=\left\{\begin{array}{cc}
\cos [\theta] & \sin [\theta] \\
-\sin [\theta] & \cos [\theta]
\end{array}\right\}\left\{\begin{array}{l}
x^{\prime} \\
y^{\prime}
\end{array}\right\}=\mathbf{R}\left\{\begin{array}{l}
x^{\prime} \\
y^{\prime}
\end{array}\right\}
$$

Where $\mathbf{R}[\theta]$ is the $2 \times 2$ rotation matrix having unit determinant. Therefore, an incident ray hitting the plate at an arbitrary position will be rotated an angle $\theta$ about the normal to the plate's normal. It is emphasized in view of the following that the matrix $\left\{\begin{array}{cc}\mathbf{R}[\theta] & \mathbf{0} \\ \mathbf{0} & \mathbf{R}[\theta]\end{array}\right\}$ that rotates both the input/output positions and simultaneously the associated ray directions will be symplectic. Following the example given in [6] and [9] we examine the effect of the flipping plate on a one-dimensional expanding field $\exp \left[-i k x^{2} /(2 R)\right]$ with radius of curvature $R$. The light ray direction of this is $\left(x^{\prime}, y^{\prime}\right)=(x / R, 0)$, where we have omitted the unimportant $z$-component. The curl of this will vanish as expected due to the curl of a field that is determined by the gradient of a potential equals zero. We now let this field pass the flipping plate with an angular twist $\theta$, after which the ray direction according to Eq. 6 becomes $\left(p_{x}{ }^{\prime}, p_{y}{ }^{\prime}\right)=(x \cos [\theta] / R, x \sin [\theta] / R)$. The curl of this is:

$$
\partial(x \sin [\theta] / R) / \partial x-\partial(x \cos [\theta] / R) / \partial y=\sin [\theta] / R,
$$


which only vanishes for the angle of rotation being zero or pi. In short, the ray plate has created a field with non-zero curl across the entire field! Such a field cannot be created as a continuous field, but the possibility of doing so in a pixelated way has been devised and experimentally proven in two ways. One way was to establish an array of Dove prisms, each of which flipped the incident ray direction the desired angle [7]; another experimental setup obtained the same pixelated filter by having two cylindrical lens arrays sharing the same focal point [7]. The outcome of these experiments are not shown here, but will later be linked with the results presented in the following.

\section{THE NON-SYMPLECTIC MATRIX FOR THE RAY-FLIPPING PLATE}

By scrutinizing the effect of the ray matrix (1) and the outcome of the ray-flipping plate (6), it is tempting to introduce a new matrix:

$$
\mathbf{M}_{\text {Flip }}=\left\{\begin{array}{cccc}
1 & 0 & 0 & 0 \\
0 & 1 & 0 & 0 \\
0 & 0 & \cos [\theta] & \sin [\theta] \\
0 & 0 & -\sin [\theta] & \cos [\theta]
\end{array}\right\} .
$$

The determinant of this matrix is unity, but as expected, it is only symplectic for flipping angles zero and pi, the latter mimicking the effect of phase conjugation.

Knowing the ray matrix, we are able to derive the Green's function that connects the field in the output plane with the field in the input plane [10]:

$$
U_{\text {out }}(\mathbf{p}) \propto \int_{-\infty}^{\infty} \int_{-\infty}^{\infty} U_{\text {in }}(\mathbf{r}) \exp \left[i \pi\left(\mathbf{r}^{t} \mathbf{B}^{-1} \mathbf{A r}-2 \mathbf{r}^{t} \mathbf{B}^{-1} \mathbf{p}+\mathbf{p}^{t} \mathbf{D} \mathbf{B}^{-1} \mathbf{p}\right)\right] d \mathbf{r} .
$$

Here the suffix "t" stands for "transpose" and the three submatrices are displayed in (1). In the above equation, we have omitted a common factor, which is of little importance in what follows.

When we analyze a general 2D optical system, the result will be analytical but usually rather lengthy. Therefore, we will provide some simple examples showing what to expect if a flipping filter is included in an optical train of elements.

In case of Fourier transforming the filter, we establish the matrix for the optical train consisting of filter with flipping angle $\theta$ - free space $f$ - lens focal length $f$ - free space $f$. Next, we derive the Green's function connecting the field in the input and output planes (9). The matrix for the Fourier transform of the filter becomes:

$$
\left\{\begin{array}{cccc}
0 & 0 & f \cos [\theta] & f \sin [\theta] \\
0 & 0 & -f \sin [\theta] & f \cos [\theta] \\
-1 / f & 0 & 0 & 0 \\
0 & -1 / f & 0 & 0
\end{array}\right\}
$$

The A submatrix being zero indicates that the output position of a ray is independent on the ray's input position, as is to be expected when Fourier transforming a field. On the other hand, in this case the rays' output position at a given position depends on both components of the ray's input slope! In the extreme, where $\theta=\pi / 2$, we have that the output $y$-position depends only on the input slope in the $x$-direction. However, for an arbitrary direction of the filter, the Fourier properties are "twisted", i.e. a given input slope gives rise to an impact at an angularly shifted position as seen by the rotation properties of the $\boldsymbol{B}$-matrix. 
In case we derive the matrix for the combination of a ray-flipping filter and an adjacent aperture, the result will depend on the order in which they appear! At first glance, this seems strange, but when diffraction at the edge of the aperture is considered, this result is properly resolved.

Let us first analyze the transformation of a system where a coherent and collimated field of width $\omega_{0}$ placed at a distance $L_{1}$ from the filter having a flipping angle $\alpha$ and a width $\omega$ observed at a varying distance, here named $L_{2}$. To highlight the effect of the filter, we have assumed the system rotationally symmetric. The matrix for the entire system becomes:

$$
\left(\begin{array}{cccc}
1-\frac{2 \mathrm{iL} 2}{k w^{2}} & 0 & \mathrm{~L} 2 \cos (a)-\frac{2 \mathrm{iL} 1 \mathrm{~L} 2}{k w^{2}}+\mathrm{L} 1 & \mathrm{~L} 2 \sin (a) \\
0 & 1-\frac{2 \mathrm{iL} 2}{k w^{2}} & -\mathrm{L} 2 \sin (a) & \mathrm{L} 2 \cos (a)-\frac{2 \mathrm{iL} 1 \mathrm{~L} 2}{k w^{2}}+\mathrm{L} 1 \\
-\frac{2 \mathrm{i}}{k w^{2}} & 0 & \cos (a)-\frac{2 \mathrm{iL} 1}{k w^{2}} & \sin (a) \\
0 & -\frac{2 \mathrm{i}}{k w^{2}} & -\sin (a) & \cos (a)-\frac{2 \mathrm{iL} 1}{k w^{2}}
\end{array}\right)
$$

The intensity distribution in the output plane becomes:

$$
\begin{gathered}
I_{\text {out }}(\mathbf{p}) \propto \int_{-\infty}^{\infty} \int_{-\infty}^{\infty} U_{\text {in }}\left(\mathbf{r}_{1}\right) U_{\text {in }} *\left(\mathbf{r}_{2}\right) \exp \left[i \pi\left(\mathbf{r}_{1}^{t} \mathbf{B}^{-1} \mathbf{A} \mathbf{r}_{1}-2 \mathbf{r}_{1}^{t} \mathbf{B}^{-1} \mathbf{p}+\mathbf{p}^{t} \mathbf{D} \mathbf{B}^{-1} \mathbf{p}\right)\right] \\
\left(\exp \left[i \pi\left(\mathbf{r}_{2}^{t} \mathbf{B}^{-1} \mathbf{A} \mathbf{r}_{2}-2 \mathbf{r}_{2}^{t} \mathbf{B}^{-1} \mathbf{p}+\mathbf{p}^{t} \mathbf{D} \mathbf{B}^{-1} \mathbf{p}\right)\right]\right) * d \mathbf{r}_{1} d \mathbf{r}_{2} .
\end{gathered}
$$

Having the ray-matrix makes us arrive at an analytical expression for the output intensity. Some illustrative examples is given in the following with the field emanating from a distance of $10 \mathrm{~mm}$ and a wavelength of 1 micron.

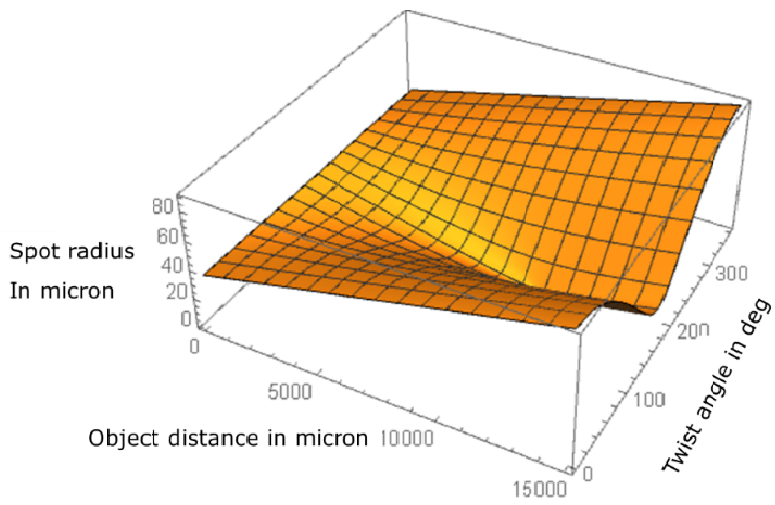

Fig. 1. Spot radius as a function of the distance from the filter and the flipping angle of the filter. The incident spot size here is 5 micron.

Fig. 1 shows how a flipping angle of $180^{\circ}$ will create a focusing of the incident field, whereas an angle of $90^{\circ}$ will cause the field to be collimated - yet twisting about the axis- as it propagates, and start to diffract at a larger distance. For flipping angles close to $0^{0}$, the field will diffract as if the plate did not exist. In the above, the aperture of the filter is large, and thus does not influence the outcome.

Fig. 2. Shows the irradiance dimeter in the observation plane for an incident spot size of 0.5 micron. As expected, an increased irradiance is observed in the far field, but still focusing exists for flipping angles close to $180^{\circ}$. 


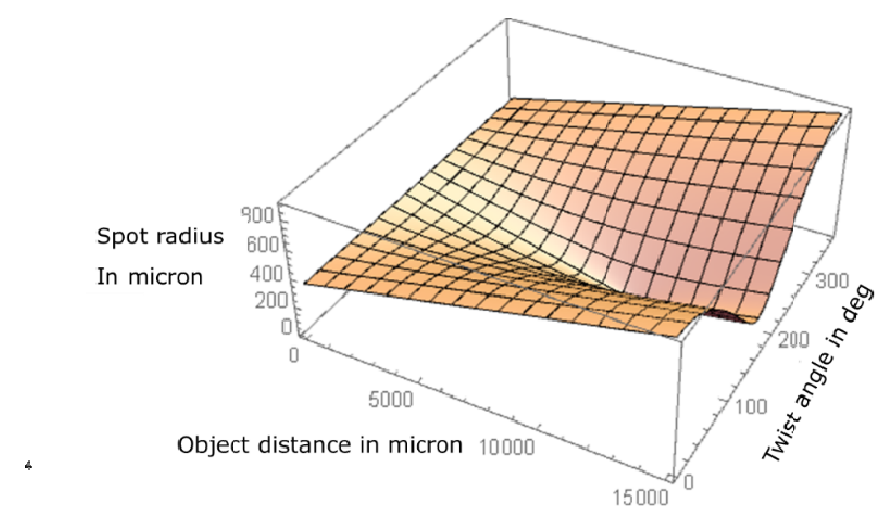

Fig. 2. Spot radius as a function of the distance from the filter and the flipping angle of the filter. The incident spot size here is .5 micron.

The last example illustrates the influence of the width of the filter.

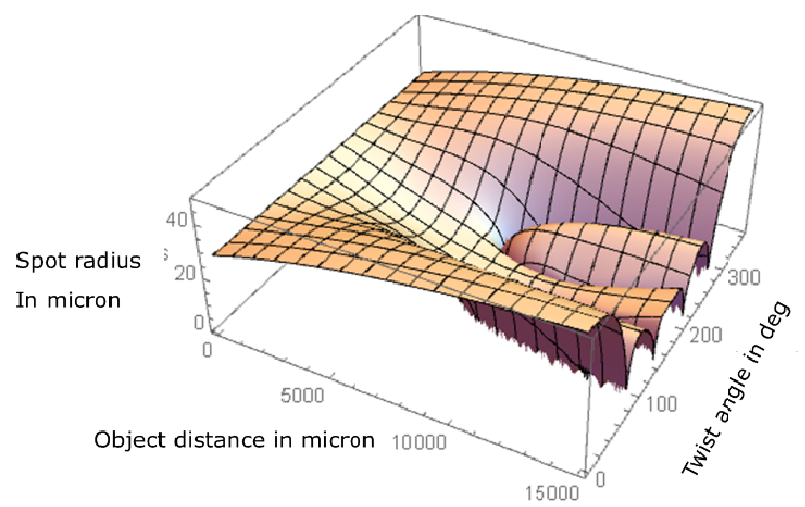

Fig. 3. Spot radius as a function of the distance from the filter and the flipping angle of the filter. The incident spot size here is 50 micron and the filter diameter is 100 micron.

It is here evident that the competition between diffraction from the filter and the flipping effect sets in.

Based on the use of ray matrices and the associated Green's function, a broad ensemble of optical systems can analytically treated. Meanwhile, it is emphasized that the theory is only valid within the Fresnel regime, usually restricting the applicability to distances separated from the filter. In this way correlation functions, phase, and incoherent light, including ordinary imaging, can be treated. 


\section{THE ROSETTE FILTER}

In like manner, as was the case for the flipping filter, an alternative filter is conceived. Whereas the previous filter changed the outgoing angle with respect to the incoming direction, the Rosette filter changes the rays azimuthally, as well, but now based on the radial position. Fig. 4 shows the proposed filter.

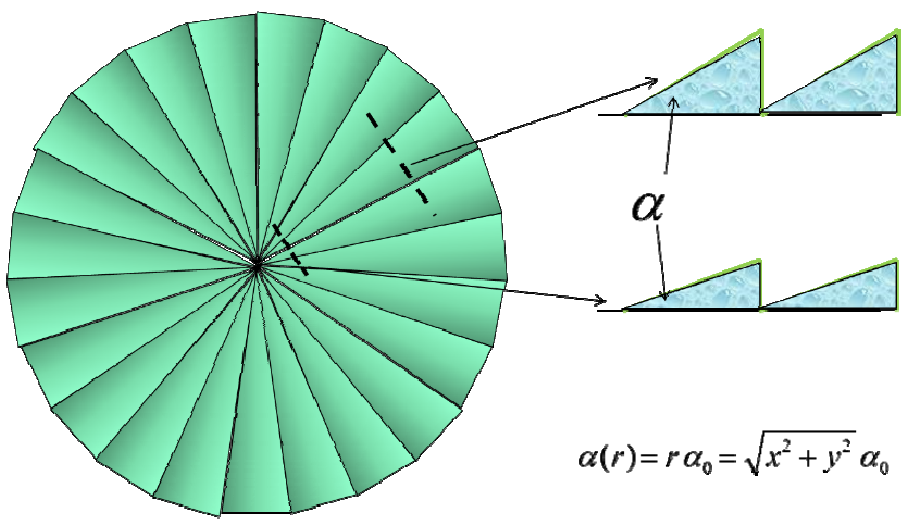

Fig.4. Rosette filter giving an angular deflection $\alpha_{0}$ proportional to the radial distance.

It is obvious that the effect of this filter appears in the $\mathbf{C}$-part of the matrix. In fact, the filter is to some extent comparable with a lens that refracts an incident ray in the plane defined by the incident ray and the normal to the lens plane. Here, we refract light perpendicular to this plane. It is emphasized that a small refraction in the radial direction is associated with the azimuthal refraction. This is not included in the following, but could be taken into account in a similar manner as in Ref. 11, giving an inhomogeneous ray matrix. Here, the matrix describing this filter consequently turns out to be:

$$
\left\{\begin{array}{l}
p_{x, \text { out }} \\
p_{y, \text { out }} \\
p_{x, \text { out }}^{\prime} \\
p^{\prime}{ }_{y, \text { out }}
\end{array}\right\}=\left\{\begin{array}{cccc}
1 & 0 & 0 & 0 \\
0 & 1 & 0 & 0 \\
0 & -(n-1) \alpha_{0} & 1 & 0 \\
(n-1) \alpha_{0} & 0 & 0 & 1
\end{array}\right\}\left\{\begin{array}{c}
x_{i n} \\
y_{i n} \\
x_{i n}^{\prime} \\
y^{\prime}{ }_{i n}
\end{array}\right\} \text { or }\left\{\begin{array}{c}
\mathbf{p} \\
\mathbf{p}^{\prime}
\end{array}\right\}\left\{\begin{array}{cc}
\hat{\mathrm{I}} & \hat{0} \\
\hat{C} & \hat{\mathrm{I}}
\end{array}\right\}\left\{\begin{array}{l}
\mathbf{r} \\
\mathbf{r}^{\prime}
\end{array}\right\}
$$

where $n$ is the refractive medium of the transparent material, A spiral phase plate has previously been described [11] where the angular displacement is inversely proportion to the radial distance, giving a forbidden region close to the center. The associated matrix was inhomogeneous due to the coordinates being included.

We will make it up to the reader to elaborate on the outcome of using this filter. Here, we will merely describe the effect of Fourier transformation. Inserting the Rosette filter in the front focal plane of a lens with focal length $f$ and observing the field in its back focal plane, we get the following matrix:

$$
\left\{\begin{array}{cccc}
0 & -f(n-1) \alpha_{0} & f & 0 \\
f(n-1) \alpha_{0} & 0 & 0 & f \\
-1 / f & 0 & 0 & 0 \\
0 & -1 / f & 0 & 0
\end{array}\right\}
$$

The off-axis elements of the $\mathbf{C}$-matrix for the original element now appears in the A-element showing that besides a Fourier transformation of the field, we now in addition have a scaling between the input- and output positions, i.e. a shift between the $\mathrm{x}$ - and $\mathrm{y}$-coordinates appear. 


\section{CONCLUSIONS}

An operational way of dealing with two optically elements that can only be implemented in discrete form, and which do not obey Fermat's principle, has been discussed. The method hinges on assigning non-symplectic ray matrices (Canonical Transform) to the element, and based on these, use the associated Green's function. A couple of examples on field propagation show good agreement with simulations with the so-called METATOY program [1].

The field having passed the filters will possess finite curl, which means that vortices will be predominant opening up for applications within optical tweezers, cell sorting and measurement involving orbital angular momentum. The main issue for future application centers on how to implement a structure inducing finite curl! If we start with the pixelated structure, a decrease in each substructure will increase the diffraction properties, believed to inevitable blur the filter's effect.

\section{ACKNOWLEDGMENT}

The author acknowledge valuable discussions with Johannes Courtial, Department of Physics \& Astronomy, University of Glasgow, UK and Miguel A. Alonso, The Institute of Optics, University of Rochester, Rochester, NY 14627, U.S.A

\section{REFERENCES}

1. J. Courtial and T. Tyc, "METATOYs and optical vortices," J. Opt. 13(11), (2011).

2. A. Koc, L. Hesselink, and H. M. Ozaktas, "Fast and accurate algorithm for the computation of complex linear canonical transforms," J. Opt. Soc. Am. A Opt. Image Sci. Vis. 27(9), 1896-1908 (2010).

3. S. A. Collins, "Lens-System Diffraction Integral Written in Terms of Matrix Optics," J. Opt. Soc. Am. 60(9), 1168- (1970).

4. A. E. Siegman, Lasers (University Science Books, 1986), 1.

5. H. T. Yura and S. G. Hanson, "Optical Beam Wave-Propagation through Complex Optical-Systems," J. Opt. Soc. Am. A-Optics Image Sci. Vis. 4(10), 1931-1948 (1987).

6. M. Blair, L. Clark, E. A. Houston, G. Smith, J. Leach, A. C. Hamilton, and J. Courtial, "Experimental demonstration of a light-ray-direction-flipping METATOY based on confocal lenticular arrays," Opt. Commun. 282(21), 4299-4302 (2009).

7. M. Blair, L. Clark, E. A. Houston, G. Smith, J. Leach, A. Hamilton, and J. Courtial, "Experimental demonstration of a light-ray-direction-flipping METATOY based on confocal lenticular arrays," (2009).

8. J. Courtial and T. Tyc, "METATOYs and optical vortices," J. Opt. 13(11), (2011).

9. M. A. Courtial, Johannes; Kirkpatrick, Blair C.; Alonso, "Imaging with complex ray-optical refractive-index interfaces between complex object and image distances," Opt.Lett. 37(4), 701-703 (2012).

10. M. B. Tatiana Alieva, "Alternative representatio of the linear canonical integral transform," Opt.Lett. 30(24), 3302 (2005).

11. M. Eggleston, T. Godat, E. Munro, M. A. Alonso, H. Shi, and M. Bhattacharya, "Ray transfer matrix for a spiral phase plate," (2013). 